\title{
Yoga Therapy for Chronic Tension-Type Headache
}

\author{
Neha Sharma, $\mathrm{PhD}^{1,4}$, PJ John, $\mathrm{PhD}^{2}$, Chandra M Sharma, $\mathrm{DM}{ }^{3} \&$ Ram Vaidya, $\mathrm{PhD}^{4}$ \\ ${ }^{1}$ NMP Medical Research Institute, Jaipur, India \\ ${ }^{2}$ University of Rajasthan, Jaipur, India \\ ${ }^{3}$ SMS Medical College \& Hospital, Jaipur, Rajasthan, India \\ ${ }^{4}$ Yog-Kulam, London, UK \\ Correspondence: Neha Sharma, 143 Loughborough Road, Leicester LE4 5LR, United Kingdom. Tel: \\ 44-744-830-7225. E-mail: drnsharma@outlook.com
}

Received: February 28, 2019 Accepted: March 19, 2019 Online Published: April 8, 2019

doi:10.5539/gjhs.v11n5p94

URL: https://doi.org/10.5539/gjhs.v11n5p94

\begin{abstract}
Objective: To evaluate the clinical efficacy of yoga therapy for Chronic Tension-Type Headache.

Method: 68 patients diagnosed with chronic tension-type headache were included in 12- week yoga therapy program. All patients completed baseline assessments before starting the intervention and at 4, 8 and 12 weeks completing the yoga therapy. Headache frequency, duration, intensity and analgesics use were recorded through headache diaries.

Result: Compared with baseline values, mean headache days reduced 51\% after 4-week of intervention which continued to reduce by $78 \%$ by end of 12 weeks. Comparing from baseline, at 12 -week, duration of each headache attack also significantly $(\mathrm{P}<0.0001)$ shortened as well as headache intensity $(\mathrm{p}<0.0001)$. Days with medication per four weeks at post intervention were lower than those at the baseline.
\end{abstract}

Conclusion: The study provided preliminary evidence that yoga therapy can be clinically useful for chronic tension-type headache. Further randomised controlled trial is needed.

\section{Clinical Trials.gov Identifier: NCT03862638}

Keywords: Yoga therapy, chronic tension type headache, frequency, analgesics use, headache, Yoga

\section{Introduction}

Chronic Tension-Type Headache (CTTH) is primary headache disorder affecting $2 \%$ of population worldwide. It evolves from episodic tension-type headache, to more frequent headaches (15 or more days a month) lasting few minutes to several days (IHS, 2013). Chronic tension headache not only imposes significant social and economic burden (WHO, 2004; Schwartz et al., 1997) but negatively impacts daily life and overall quality of life (Linde et al. 2012; Stovner et al., 2007). Chronic tension headaches often lead to overuse of analgesic and change to rebound headaches. Due to associated risk factors, analgesics overuse and comorbid psychiatric issues, Chronic tension headache become more difficult to manage in primary care (Holroyd et al., 2000). Guidelines recommend antidepressants such as amitriptyline (Bendtsen et al., 1996). However, recent trials reported little or no improvements with amitriptyline in CCTH (Pfaffenrath et al., 1994, Gobel, 1994).

In addition to, or instead of drug therapy, behavioural interventions such as relaxation or biofeedback have been shown to be beneficial (Holroyd et al., 2005; Holroyd et al., 2001; Holroyd et al., 2002). However, additional effective intervention tools with self-management, feasibility and tolerability are desirable. Yoga therapy could possibly be one approach to manage CCTH.

Yoga therapy is a combination of physical postures, breathing exercises, guided meditation, relaxation, and cleansing practices (Kriyas). Yoga has been reported as a safe and cost-effective intervention for managing pain (Nespore, 1991; Evan et al., 2008; Sharma et al., 2013; Büssing et al., 2012; Latha et al., 1987). Recent evidences interlink the physical and psychological benefits of yoga through the mechanisms of down-regulation of the hypothalamic-pituitary-adrenal axis and the sympathetic nervous system (Damodaran et al., 2012; Vural et al., 2014; Bae et al., 2013). Benefit of yoga in reducing headache frequency, severity and duration of migraine without aura (Wells et al., 2014; Peter et al., 2013; John et al., 2007) and tension type headache (Menon, 2013) has been 
documented. One study reported highly significant reduction in severity, duration and frequency of headaches in CCTH with raja yoga meditation (Kirn et al., 2014).

Effectiveness of yoga therapy in the management of chronic tension type headache is limited. Therefore, present study was undertaken to assess the effect of yoga as an intervention in chronic tension type headache.

\section{Methods}

Study used pre-post design to assess the clinical efficacy of yoga therapy in reducing the incidence, duration and severity of pain in CCTH patients.

\subsection{Study Setting and Participants}

The study was carried out in outpatient clinic of NMP medical Research Institute, India, from April 2010, to December 2012. The study protocol was approved by the Institutional Ethics Committee (Approval no. 20897)

Initially, patients were screened and recruited by the neurologists in the clinic. Patients with chronic tension-type headache diagnosed by criteria of International Headache Society (IHS, 2004) having not received any treatment in the previous week, besides symptomatic medication. Eligible patients aged 18-55 years, with CTTH for more than a year, suffering from TTH for at least 15 days a month during the previous 3 months, and agreed to provide informed consent were included.

Patients were excluded if having secondary headache disorder or systemic disorders; pregnancy; and patients with psychiatric conditions, drug and substance abuse, having any other complementary and alternative medicines.

\subsection{Outcome Measures}

Following the inclusion and exclusion criteria, neurologist referred eligible participants to yoga intervention. Those who were eligible and willing to participate were assessed and followed by an independent physician during the study period. Study protocol was explained to participants, and written informed consents were obtained after a detailed explanation of the study purpose and methods.

Baseline assessment included a detailed history, physical examination, and collection of baseline data. All patients filled in headache questionnaire at baseline and 4, 8, and 12 weeks of yoga intervention. As the main outcome measures, the headache diaries were given to record headache score on visual analogue scale from zero (no pain) to 10 (most severe pain), duration of each attack (in hours), the number of days on which headaches occurred per four weeks and analgesics use per four weeks.

\subsection{Intervention}

Completed baseline assessment, 12-week yoga therapy sessions were started. Participants were asked to attend group therapy session thrice a week alternate days and remaining days to practice at home. Each session lasted for 60 minutes consisting physical posture, breathing exercise, relaxation techniques, guided meditation, chants and combination techniques for groups sessions. Home practice were given as handouts to chart practices for each participant to follow at the same way.

\subsection{Statistical Analysis}

Statistical methods used included paired t-tests for comparison of mean values. All analyses were carried out using SPSS Statistics 19.0. $\mathrm{P}<0.05$ was considered statistically significant.

\section{Results}

A total of seventy patients with chronic tension-type headache, mean age of 34.5, who met the inclusion criteria, were included (Figure 1). Two patients did not complete therapy. One moved out of city and another started anti-depressant.

The baseline characteristics are shown in Table 1. Table 2 summarizes the results for medical outcomes for patients completing 12 weeks of intervention; at baseline and 4, 8, and 12 weeks post intervention.

There were significant changes over time after starting yoga therapy. In the primary outcome analysis, mean headache frequency was significantly lower in the group. Scores fell by $51 \% \%$ at the end of 4 -week intervention. At the end of 12 -week therapy, frequency reduced $78 \%$ comparing from baseline. Effect of yoga therapy was consistent by 12 -week. Duration of each attack also significantly $(\mathrm{P}<0.0001)$ 


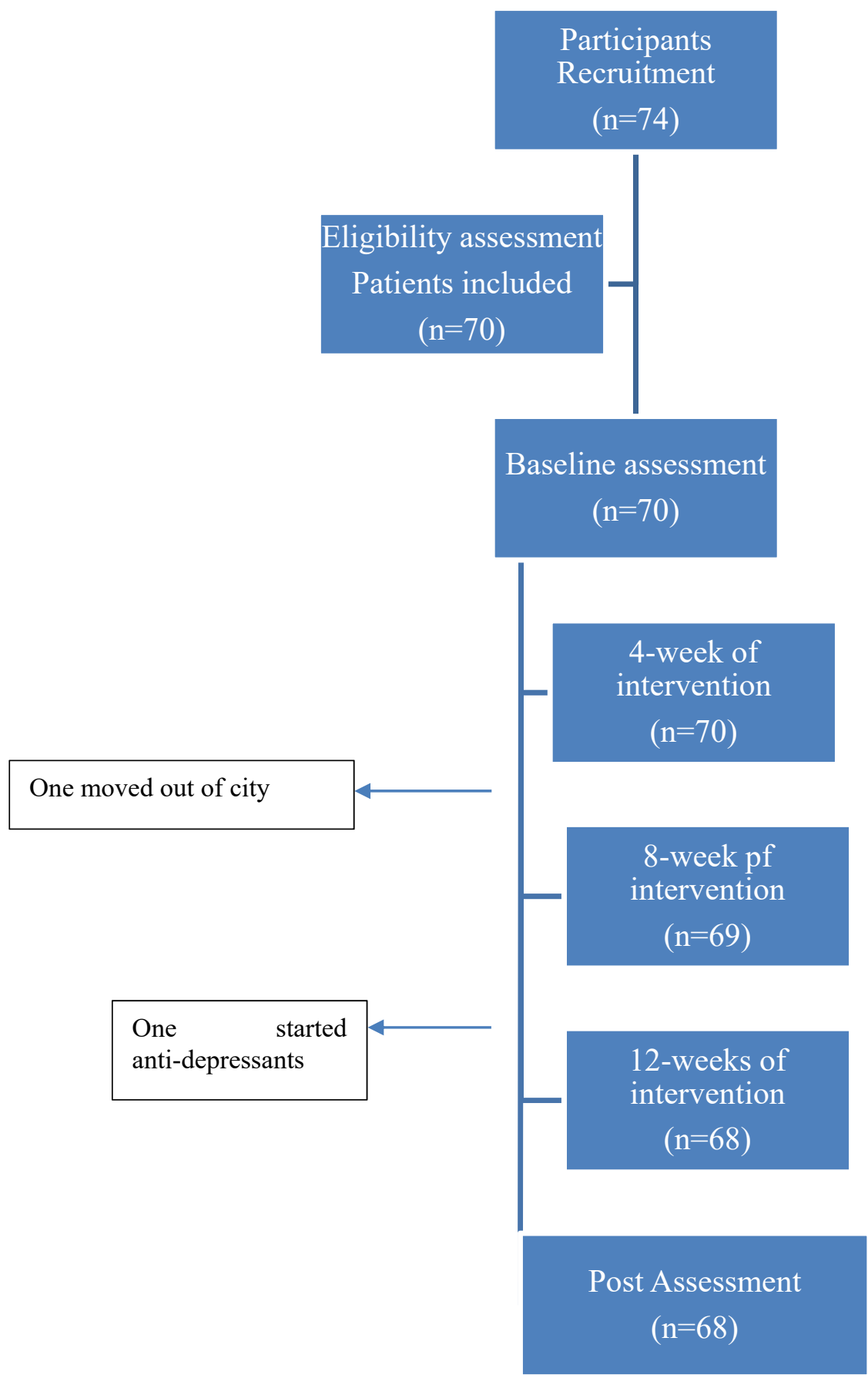

Figure 1 . Flow chart of entry and discontinuation by patients during the study 
Table 1. Baseline characteristics of Patients

\begin{tabular}{ll}
\hline All patients & $\mathrm{N}=68$ \\
\hline Gender, Male (\%) & $23(33.8 \%)$ \\
Age mean (SD) & $34.5(11.1)$ \\
Years having headache, mean (SD) & $7.9(3.1)$ \\
Days with headache per four weeks mean (SD) & $18.7(2.7)$ \\
Duration of each attack (hours)mean (SD) & $13.0(4.8)$ \\
Headache score mean (SD) & $6.4(0.7)$ \\
Days with medication per four weeks mean (SD) & $13.7(3.8)$ \\
\hline
\end{tabular}

Table 2. Change over 4, 8 and 12 weeks of yoga therapy

Outcome measures- Mean (SE)

\begin{tabular}{lllll}
\hline & Baseline & 4 weeks & 8 weeks & 12 weeks \\
\hline Headache score & $6.4(0.09)$ & $4.1(0.08)^{*}$ & $3.4(0.06)^{*}$ & $3.5(0.06)$ \\
Duration of each attack (hours) & $13.0(3.5)$ & $6.9(0.59)^{*}$ & $5.4(0.06)^{*}$ & $5.1(0.07)$ \\
Days with headache/four weeks & $18.7(0.3)$ & $9.11(0.2)^{*}$ & $4.9(0.1)^{*}$ & $4.0(0.09)^{*}$ \\
Days with medication/ four weeks & $13.7(0.46)$ & $8.5(0.20)^{*}$ & $4.6(0.11)^{*}$ & $3.7(0.11)^{*}$
\end{tabular}

Paired t-tests: $* \mathrm{P}<0.0001$.

\section{Discussion}

To best of our knowledge, this is the first clinical study to assess the key variables of headache in patients with chronic tension-type headache given yoga therapy. Results indicate that yoga therapy was found to have a beneficial effect with significant reduction in headache frequency, intensity, duration of pain and medication score.

Despite this fact that yoga cannot prevent or treat diseases itself, it relaxes muscles, regulate blood circulation and help patients feeling better in general. Yoga has been widely used for treating medical conditions, and special sets of practices used in combinations for therapeutic purpose defines yoga therapy. Our study used the specific yoga sets and combinations including physical posture, breathing and relaxation practices, which had not been studied previously in CCTH.

Yoga practices helps to reduce sympathetic nervous system activity (Streeter et al., 2012) whereas regulated breath work (pranayama) balances the autonomic nervous system (Telles et al., 1993; Bhattacharya et al., 2002) and has a powerful influence on stress release (Chong et al., 2011), as a significant risk factors of tension type headache. This explains the possible mechanism of yoga efficacy in our patients.

Yoga therapy produced clinically significant improvement compared to the baseline for each 4 weeks of intervals assessed. Symptoms of chronic tension-type headache abated significantly, and the effects were sustained through the period of 12 weeks. Methodological strengths of our study include a large sample size and high follow-up rates. There had been no safety concerns raised nor any issue of tolerability. Patients recorded the use of analgesics for headache during the study, which were lower after therapy, indicating that the superior results were not due to influence of effective cointerventions.

Study had major limitations of no having comparison group, randomisation and follow up data. Results thus cannot generalised to CCTH. Further studies are required using randomised controlled settings with. Longer follow up periods. However, present study does provide preliminary support the clinical efficacy of yoga therapy in $\mathrm{CCTH}$.

\section{Acknowledgements}

Present study was conducted and supported by the NMP Medical Research Institute. The authors thank research team, yoga therapists and patients who participated in this study.

\section{Competing Interests Statement}

All the authors declare that they do not have no financial or personal interest, relationship or ties with people or 
organizations that can inappropriately influence research work; there is no interest of any kind, professional or personal of any nature or kind in any product, service, and/or company that could be the review, results or data presented in the paper.

\section{References}

Bae, Y., \& Park, Y. (2013). The effect of relaxation exercises for the masticator muscles on temporomandibular joint dysfunction (TMD). Journal of physical therapy science, 25(5), 583-586. https://doi.org/10.1589/jpts.25.583

Bendtsen, L., Jensen, R., \& Olesen, J. (1996). A non-selective (amitriptyline), but not a selective (citalopram), serotonin reuptake inhibitor is effective in the prophylactic treatment of chronic tension-type headache. Journal of Neurology, Neurosurgery \& Psychiatry, 61(3), 285-290. https://doi.org/10.1136/jnnp.61.3.285

Bhattacharya, S., Pandey, U., \& Verma, N. (2002). Improvement in oxidative status with yogic breathing in young healthy males. Indian J Physiol Pharmacol, 46(3), 349-54.

Büssing, A., Ostermann, T., Lüdtke, R., \& Michalsen, A. (2012). Effects of yoga interventions on pain and pain-associated disability: a meta-analysis. The Journal of Pain, 13(1), 1-9. https://doi.org/10.1016/j.jpain.2011.10.001

Chong, C. S., Tsunaka, M., \& Chan, E. P. (2011). Effects of yoga on stress management in healthy adults: a systematic review. Alternative therapies in health and medicine, 17(1), 32-38.

Damodaran, A., Malathi, A., Patil, N., Shah, N., \& Marathe, S. (2002). Therapeutic potential of yoga practices in modifying cardiovascular risk profile in middle aged men and women. The Journal of the Association of Physicians of India, 50(5), 633-640.

Evans, S., Subramanian, S., \& Sternlieb, B. (2008). Yoga as treatment for chronic pain conditions: a literature review. International Journal on Disability and Human Development, 7(1), 25-32. https://doi.org/10.1515/IJDHD.2008.7.1.25

Göbel, H., Hamouz, V., Hansen, C., Heininger, K., Hirsch, S., Lindner, V., ... \& Soyka, D. (1994). Chronic tension-type headache: amitriptyline reduces clinical headache-duration and experimental pain sensitivity but does not alter pericranial muscle activity readings. Pain, 59(2), 241-249. https://doi.org/10.1016/0304-3959(94)90077-9

Holroyd, K. A., Martin, P. R., \& Nash, J. M. (2005) Psychological treatments of tension-type headache. In J. Olesen, P. J. Goadsby, N. Ramadan, P. Tfelt-Hansen, \& K. M. Welch, (Eds.), The Headaches (pp. 711-719). Philadelphia: Lippincott Williams Wilkins.

Holroyd, K. A. (2002) Assessment and psychological management of recurrent headache disorders. J Consult Clin Psychol, 70, 656-677. https://doi.org/10.1037/0022-006X.70.3.656

Holroyd, K. A., O'donnell, F. J., Stensland, M., Lipchik, G. L., Cordingley, G. E., \& Carlson, B. W. (2001). Management of chronic tension-type headache with tricyclic antidepressant medication, stress management therapy, and their combination: a randomized controlled trial. Jama, 285(17), 2208-2215. https://doi.org/10.1001/jama.285.17.2208

Holroyd, K. A., Stensland, M., Lipchik, G. L., Hill, K. R., O'Donnell, F. S., \& Cordingley, G. (2000). Psychosocial correlates and impact of chronic tension-type headaches. Headache: The Journal of Head and Face Pain, 40(1), 3-16. https://doi.org/10.1046/j.1526-4610.2000.00001.x

John, P. J., Sharma, N., Sharma, C. M., \& Kankane, A. (2007). Effectiveness of yoga therapy in the treatment of migraine without aura: a randomized controlled trial. Headache: The Journal of Head and Face Pain, 47(5), 654-661. https://doi.org/10.1111/j.1526-4610.2007.00789.x

Kiran, Girgla, K. K., Chalana, H., \& Singh, H. (2014). Effect of rajyoga meditation on chronic tension headache. Indian J Physiol Pharmacol, 58(2), 157-61.

Latha, M., \& Kaliappan, K. V. (1987). The efficacy of yoga therapy in the treatment of migraine and tension headaches. J Indian Acad Appl Psychol, 13, 95-100.

Linde, M., Gustavsson, A., Stovner, L. J., Steiner, T. J., Barré, J., Katsarava, Z., ... \& Andrée, C. (2012). The cost of headache disorders in Europe: the Eurolight project. Eur $J$ Neurol, 19, 703-711. https://doi.org/10.1111/j.1468-1331.2011.03612.x

Menon, B., Satyanand, V., \& Karishma, P. H. (2013). Effects of yoga on tension headache. J Dr NTR Univ Health 
Sci, 2, 167-70. https://doi.org/10.4103/2277-8632.117181

Nespor, K. (1991). Pain management and yoga. Int J Psychosom, 38, 76-81.

Olesen, J., \& Steiner, T. J. (2004). The International classification of headache disorders, 2nd edn (ICDH-II).

Pfaffenrath, V., Diener, H. C., Isler, H., Meyer, C., Scholz, E., Taneri, Z., ... \& Fischer, W. (1994). Efficacy and tolerability of amitriptylinoxide in the treatment of chronic tension-type headache: a multi-centre controlled study. Cephalalgia, 14(2), 149-155. https://doi.org/10.1046/j.1468-2982.1994.1402149.x

Preter, M., \& Lieblich, S. (2013). Complementary and alternative medicine (CAM) approaches to headache. In M. W. Green, \& P. R. Muskin (Eds.), the neuropsychiatry of headache (pp. 131-148). New York: Cambridge University Press.

Schwartz, B. S., Stewart, W. F., \& Lipton, R. B. (1997). Lost workdays and decreased work effectiveness associated with headache in the workplace. Journal of occupational and environmental medicine, 39(4), 320-327. https://doi.org/10.1097/00043764-199704000-00009

Sharma, N., Singhal, S., Singh, A. P., \& Sharma, C. M. (2013). Effectiveness of integrated yoga therapy in treatment of chronic migraine: randomized controlled trial. The journal of headache and pain, 14(1), P116. https://doi.org/10.1186/1129-2377-14-S1-P116

Stovner, L. J., Hagen, K., Jensen, R., Katsarava, Z., Lipton, R. B., Scher, A. I., ... \& Zwart, J. A. (2007). The global burden of headache: a documentation of headache prevalence and disability worldwide. Cephalalgia, 27(3), 193-210. https://doi.org/10.1111/j.1468-2982.2007.01288.x

Streeter, C. C., Gerbarg, P. L., Saper, R. B., Ciraulo, D. A., \& Brown, R. P. (2012). Effects of yoga on the autonomic nervous system, gamma-aminobutyric-acid, and allostasis in epilepsy, depression, and $\begin{array}{lllll}\text { post-traumatic stress } & \text { disorder. }\end{array}$ https://doi.org/10.1016/j.mehy.2012.01.021

Telles, S., Joseph, C., Venkatesh, S., \& Desiraju, T. (1993). Alterations of auditory middle latency evoked potentials during yogic consciously regulated breathing and attentive state of mind. International Journal of Psychophysiology, 14(3), 189-198. https://doi.org/10.1016/0167-8760(93)90033-L

Vural, M., Berkol, T. D., Erdogdu, Z., Pekedis, K., Kuçukserat, B., \& Aksoy, C. (2014). Evaluation of the effectiveness of an aerobic exercise program and the personality characteristics of patients with fibromyalgia syndrome: a pilot study. Journal of physical therapy science, 26(10), 1561-1565. https://doi.org/10.1589/jpts.26.1561

Wells, R. E., Burch, R., Paulsen, R. H., Wayne, P. M., Houle, T. T., \& Loder, E. (2014). Meditation for migraines: a pilot randomized controlled trial. Headache: The Journal of Head and Face Pain, 54(9), 1484-1495. https://doi.org/10.1111/head.12420

World Health Organization [WHO]. (2004). Retrieved from http://www.who.int/healthinfo/global_burden_disease/estimates_regional/en/index.html. Geneva

\section{Copyrights}

Copyright for this article is retained by the author(s), with first publication rights granted to the journal.

This is an open-access article distributed under the terms and conditions of the Creative Commons Attribution license (http://creativecommons.org/licenses/by/4.0/). 